\title{
Measurement of hair iron concentration as a marker of body iron content
}

\author{
CEM SAHIN $^{1}{ }^{2}$ CIGDEM PALA $^{2}$, LEYLAGUL KAYNAR $^{2}$, YASEMIN ALTUNER TORUN ${ }^{3}$, \\ AYSUN CETIN $^{4}$, FATIH KURNAZ ${ }^{2}$, SERDAR SIVGIN ${ }^{2}$ and FATIH SERDAR SAHIN ${ }^{5}$ \\ ${ }^{1}$ Department of Internal Medicine, Faculty of Medicine, Muğla Sitki Kocman University, Orhaniye, Muğla 48000; \\ ${ }^{2}$ Department of Hematology, Faculty of Medicine, Erciyes University, Melikgazi, Kayseri 38039; \\ ${ }^{3}$ Department of Pediatric Hematology, Education and Research Hospital, Kayseri, Kayseri 38010; \\ ${ }^{4}$ Department of Biochemistry; ${ }^{5}$ Graduate School of Health Sciences Stem Cell Sciences, \\ Faculty of Medicine, Erciyes University, Melikgazi, Kayseri 38039, Turkey
}

Received October 12, 2014; Accepted November 10, 2014

DOI: $10.3892 /$ br.2015.419

\begin{abstract}
The aim of the present study was to define the possible association between blood parameters and hair iron concentration in patient groups showing a difference in body iron content. The study population comprised subjects with iron deficiency anaemia and transfusion-related anaemia with different body iron contents and a healthy control group. All the cases included in the study were examined with respect to hair iron concentration, serum iron, total iron-binding capacity (TIBC), transferrin saturation and erythrocyte markers in the total blood count with ferritin values. Differences in hair iron concentration were evaluated between the groups. Correlation analysis was applied to define the association between the laboratory values used as markers of body iron content and hair iron concentration. A statistically significant difference was determined in hair iron ${ }^{56} \mathrm{Fe}$ and ${ }^{57} \mathrm{Fe}$ concentrations between the group with transfusion-related anaemia, the iron deficiency anaemia group and the healthy control group $(\mathrm{P}<0.001)$. In addition, a positive correlation was determined between hair iron ${ }^{56} \mathrm{Fe}$ and ${ }^{57} \mathrm{Fe}$ concentrations and serum iron, ferritin level, transferrin saturation, mean erythrocyte volume and mean erythrocyte haemoglobin values and a negative correlation with TIBC. In conclusion, the results of the present study showed a statistically significant difference in the hair iron concentrations of the patient groups with different body iron content and these values were correlated to the laboratory markers of body iron content.
\end{abstract}

Correspondence to: Dr Cem Sahin, Department of Internal Medicine, Faculty of Medicine, Muğla Sitkı Kocman University, Haluk Özsoy Street, Orhaniye, Muğla 48000, Turkey

E-mail: cemsahin@mu.edu.tr

Key words: hair iron concentration, iron deficiency anaemia, transfusion-related anaemia

\section{Introduction}

Iron, which is one of the most important essential elements for the human organism, has a role as a co-factor in several vital metabolic reactions. Iron deficiency is the most commonly observed metabolic dysfunction, as iron is not only associated with haemoglobin and myoglobin functions, but simultaneously acts in various stages correlated with protection and obtaining energy (1).

As well as iron deficiency, excessive iron in the body is a base for metabolic impairments. The accumulation of iron in tissues and organs throughout treatment in myelodysplastic syndrome, and $\beta$ thalassemia patients who require regular blood transfusions, causes tissue damage (2). The iron accumulation observed in the liver, myocardium, the pancreas, hypophysis and joints, is often together with loss of organ function in advanced stages and leads to life-threatening complications (3).

Various tests are currently used to show the amount of body iron content, including invasive laboratory tests. As they can be applied in the majority of centres and are relatively cheap, the total blood count, serum iron concentration, total iron-binding capacity (TIBC), transferrin saturation percentage (TS) and serum ferritin level form the basis of these tests. Although their clinical use remains limited, advanced tests showing iron deficiency at a cellular level and serum-free transferrin receptor level, which is not affected by the acute phase response, are used for research. The best clinical test showing iron status is the invasive method of bone marrow aspiration stained with Prussian blue. In addition, there are other tests, including magnetic resonance imaging and organ needle biopsy, which are used to show iron accumulation in the organs (4).

Previous studies have shown that hair mineral analysis can be used as a good marker of body mineral levels. However, there are extremely limited studies associated with hair iron concentration used as a marker of body iron content. The limited number of studies has not shown a clear association and have emphasized the requirement for more research.

In the present study, whether or not hair iron concentration can be used as a marker of body iron content was investigated. 
In this context, it was aimed to define the possible association between blood parameters and hair iron concentration in patient groups with different body iron contents.

\section{Materials and methods}

Study subjects. The study population comprised patients with iron deficiency anaemia and transfusion-related anaemia that showed a difference in body iron content, who were being followed up in the Haematology Department of Internal Medicine at Erciyes University Medical Faculty and Kayseri Training and Research Hospital (Kayseri, Turkey) between September 2010 and October 2011. The control group was formed of age and gender-matched subjects with no history of underlying disease. The transfusion-related anaemia group, with an excessive amount of body iron, comprised of 20 patients diagnosed with myelodysplastic syndrome or $\beta$ thalassemia from erythrocyte suspension of $\geq 30$ units. The iron deficiency anaemia group comprised of 25 patients and the control group comprised of 21 healthy subjects were evaluated as within the reference values of TS and ferritin levels. Any cases who were pregnant; had a history of acute or chronic inflammatory disease, malignant disease, infection or renal failure; had any underlying disease, such as malabsorption syndrome, which affected serum iron and ferritin levels; or any disease associated with the hair or scalp that affected the hair iron concentration, were excluded from the study.

Serum iron, TIBC, TS, ferritin and hair iron concentration were examined in all the cases together with haemoglobin, haematocrit and erythrocyte markers, such as mean erythrocyte volume (MCV), mean erythrocyte haemoglobin $(\mathrm{MCH})$, $\mathrm{MCH}$ concentration (MCHC) and erythrocyte distribution width (RDW). TS was calculated using the formula of serum iron / iron-binding capacity x 100. The diagnosis of iron deficiency anaemia was made according to the World Health Organization criteria (5). Haemoglobin was accepted as $<13 \mathrm{~g} / \mathrm{dl}$ in males and $<12 \mathrm{~g} / \mathrm{dl}$ in females. Iron deficiency anaemia cases were classified according to reference intervals, as serum iron low, TIBC high; transferrin saturation $<15 \%$ and serum ferritin $<15 \mathrm{ng} / \mathrm{dl}$.

Blood sampling. Fasting blood samples were taken in the morning. Full blood count was assayed using an automatic electronic full blood count analyser (Siemens Advia 2120; Siemens Healthcare Diagnostics, Deerfield, IL, USA), serum iron and iron-binding capacity using a biochemical analyser (Siemens Advia 1800 Chemistry System; Siemens Healthcare Diagnostics) and ferritin using a hormone analyser ADVIA Centaur XP (Siemens Healthcare Diagnostics, Deerfield, IL, USA).

Hair sampling. Prior to the hair sampling process, no history of cosmetic hair application, such as lightening, dyeing or perm, was found in any of the cases. The hair samples in the patient and control groups were obtained with stainless steel scissors from the occipital area as far as the proximal section. Each sample collected was stored in an autosealable polythene bag and washed sequentially with acetone, deionized water and acetone, using three successive portions. Subsequently, the samples were dried in an oven at $80^{\circ} \mathrm{C}$. Each hair sample, weighing $250 \mathrm{mg}$, was transferred into a digestion vessel of $10 \mathrm{ml}$ capacity and concentrated nitric acid $(5 \mathrm{ml})$ was pipetted into the vessel. The samples were diluted with $65 \%$ (v/v) nitric oxide in a microwave oven (Berghof Speedwave, Eningen, Germany). Analytic techniques were applied with Agilent 7500a Inductively Coupled Mass Spectrometer (Agilent Technologies, Tokyo, Japan). Digestion solutions were diluted and analyzed on an Agilent 7500a series ICP/MS. For the quality control, the concentration of internal standards $200 \mathrm{ppb}(9 \mathrm{Be}, 45 \mathrm{Sc}, 103 \mathrm{Rh}, 208 \mathrm{Bi})$ and reference materials were run together with the samples and before the analyses tune parameters were cotrolled. To obtain the detection limit of each element the axis cut point of the calibration line was used. It was also important to ensure the linear rank of the methodology analyzing different standards with low and high known concentrations of each element $(0,1,5,10,20$, 30,40 and $50 \mathrm{ppb}$ ). At least five different reference materials covering all the elements in the study were used. Duplicate samples were also used in order to determine precision of the analysis. For the iron element a minimum of three standards were used to cover the analytical working range of the instrument. Ultrapure water was used to prepare calibration standards and blanks, and three replicate determinations were performed for each sample. From all the sample results, a reagent blank was subtracted. Detection limits were calculated as three times the standard deviation for the reagent blanks. LODs value (in microgram per milliliter) was $0.0008(\mathrm{Fe})$. Hair concentrations were evaluated as micrograms per gram.

Statistical analysis. Data were analysed using SPSS v20 software statistical program (IBM Corp, Armonk, NY, USA). In the evaluation of statistical differences between groups of numerical values with normal distribution, one-way analysis of variance was used. In the statistical evaluation between groups of parameters such as age, haemoglobin, $\mathrm{MCV}, \mathrm{MCH}, \mathrm{MCHC}$, RDW, serum iron, hair iron levels and TS, which did not return to normal distribution despite logarithmic transformation, the Kruskal-Wallis test was used. In all the statistical tests, a value of $\mathrm{P}<0.05$ was considered to indicate a statistically significant difference.

\section{Results}

Patient characteristics. The mean age was 33 (range, 14-48 years) in the iron deficiency anaemia group, 22 (range, 15-75 years) in the transfusion-related anaemia group and 28 (range, 13-42 years) in the control group. The degree of statistical significance of these findings together with the haematological and biochemical findings of the cases is shown in Table I. According to this, a statistically significant difference was found between the groups in all parameters except for age (Table I).

Hair iron concentration. The mean hair iron ${ }^{56} \mathrm{Fe}$ and ${ }^{57} \mathrm{Fe}$ concentrations of the iron deficiency group were measured as 5.08 and $6.03 \mu \mathrm{g} / \mathrm{g}$, respectively, and in the transfusion-related anaemia group these values were 28.88 and $29.44 \mu \mathrm{g} / \mathrm{g}$, respectively. In the control group, the mean hair iron ${ }^{56} \mathrm{Fe}$ and ${ }^{57} \mathrm{Fe}$ concentrations were measured as 12.01 and $17.62 \mu \mathrm{g} / \mathrm{g}$, respectively. The highest hair iron concentration of the ferritin value 
Table I. Results of hair iron concentration and laboratory tests of the groups.

\begin{tabular}{|c|c|c|c|c|}
\hline Parameters & Control & Iron deficiency anaemia & Transfusion-related anaemia & P-value \\
\hline Age, years (range) ${ }^{a}$ & $28(23-30)$ & $33(23.5-42)$ & $22(19-31)$ & 0.215 \\
\hline Haemoglobin, $\mathrm{g} / \mathrm{dl}^{\mathrm{a}}$ & $13.61 \pm 1.15^{\mathrm{b}}$ & $8.08 \pm 1.55^{\mathrm{c}}$ & $9.39 \pm 0.89^{d}$ & $<0.001$ \\
\hline Haematocrit, $\%^{\mathrm{e}}$ & $38.95 \pm 3.57^{\mathrm{b}}$ & $27.17 \pm 3.71^{\mathrm{c}}$ & $28.81 \pm 2.60^{c}$ & $<0.001$ \\
\hline $\mathrm{MCH}, \mathrm{pg}^{\mathrm{a}}$ & $28.87 \pm 4.07^{\mathrm{b}}$ & $18.97 \pm 3.27^{\mathrm{c}}$ & $27.83 \pm 2.53^{b}$ & $<0.001$ \\
\hline $\mathrm{MCHC}, \mathrm{g} / \mathrm{dl}^{\mathrm{a}}$ & $34.31 \pm 1.93^{\mathrm{b}}$ & $29.67 \pm 2.52^{c}$ & $32.58 \pm 1.49^{\mathrm{d}}$ & $<0.001$ \\
\hline $\mathrm{MCV}, \mathrm{fl}^{\mathrm{a}}$ (range) & $86.7^{\mathrm{b}}(83.5-88.5)$ & $64.5^{\mathrm{c}}(57.7-71.7)$ & $84.5^{\mathrm{b}}(82.5-86.4)$ & $<0.001$ \\
\hline RDW, \%a (range) & $13.1^{\mathrm{b}}(12.4-14.3)$ & $17.1^{\mathrm{c}}(16.5-17.4)$ & $18.4^{\mathrm{c}}(16-22.6)$ & $<0.001$ \\
\hline Serum iron, $\mu \mathrm{g} / \mathrm{dl}^{\mathrm{a}}$ (range) & $87^{\mathrm{b}}(46-99)$ & $9^{c}(7-13.7)$ & $189^{\mathrm{d}}(159-251)$ & $<0.001$ \\
\hline TIBC,$\mu \mathrm{mol} / 1^{\mathrm{a}}$ (range) & $300^{\mathrm{b}}(263-343)$ & $449^{c}(441-467)$ & $88.5^{\mathrm{d}}(53.5-193.5)$ & $<0.001$ \\
\hline $\mathrm{TS}, \%$ (range) & $27.2^{\mathrm{b}}(0.14-0.32)$ & $1.96^{\mathrm{c}}(0.01-0.03)$ & $279.5^{\mathrm{d}}(0.9-4.1)$ & $<0.001$ \\
\hline Ferritin, ng/ml ${ }^{\mathrm{a}}$ (range) & $32.8^{\mathrm{b}}(25.3-44.2)$ & $3.45^{\mathrm{c}}(1.8-5.9)$ & $1598^{\mathrm{d}}(1162-2508)$ & $<0.001$ \\
\hline Hair iron ${ }^{56} \mathrm{Fe}, \mu \mathrm{g} / \mathrm{g}^{\mathrm{a}}$ (range) & $12.01^{\mathrm{b}}(4.24-16.17)$ & $5.08^{\mathrm{c}}(0.77-10.87$ & $28.88^{\mathrm{d}}(21.45-89.41)$ & $<0.001$ \\
\hline Hair iron ${ }^{57} \mathrm{Fe}, \mu \mathrm{g} / \mathrm{g}^{\mathrm{a}}$ (range) & $17.62^{\mathrm{b}}(10.28-26.80)$ & $6.03^{\mathrm{c}}(1.26-9.65)$ & $29.44^{\mathrm{d}}(20.69-69.33)$ & $<0.001$ \\
\hline
\end{tabular}

${ }^{a}$ Median values; ${ }^{b, c, d}$ statistical significance between groups in the same row; ${ }^{e}$ mean values. $\mathrm{MCH}$, mean erythrocyte haemoglobin; MCHC, mean erythrocyte haemoglobin concentration; MCV, mean erythrocyte volume; RDW, erythrocyte distribution width; TIBC, total iron-binding capacity; TS, transferrin saturation percentage.

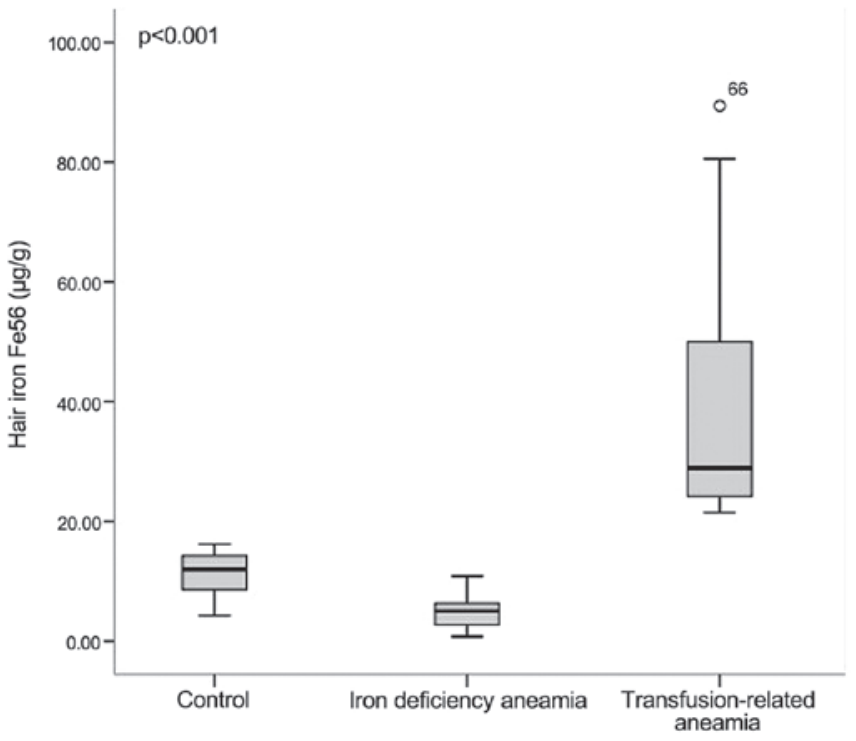

Figure 1. Results of hair iron ${ }^{56} \mathrm{Fe}$ ppm concentration in the groups.

$(89.41 \mu \mathrm{g} / \mathrm{g})$ was observed in transfusion-related anaemia patients, whereas the lowest hair iron concentration $(0.77 \mu \mathrm{g} / \mathrm{g})$ was determined in the iron deficiency anaemia group. The differences between the three groups with respect to hair iron ${ }^{56} \mathrm{Fe}$ and ${ }^{57} \mathrm{Fe}$ concentrations were found to be statistically significant (Figs. 1 and 2).

Statistical analysis. Correlation analysis applied to the parameters showed a strong positive correlation between hair iron ${ }^{56} \mathrm{Fe}$ and ${ }^{57} \mathrm{Fe}(\mathrm{r}=0.776, \mathrm{P}<0.001)$. A statistically significant positive correlation was determined between hair iron ${ }^{56} \mathrm{Fe}$ and ${ }^{57} \mathrm{Fe}$ concentrations and serum iron, ferritin level, transferrin saturation, MCV and $\mathrm{MCH}$ values. A statistically significant negative correlation was determined between hair iron ${ }^{56} \mathrm{Fe}$

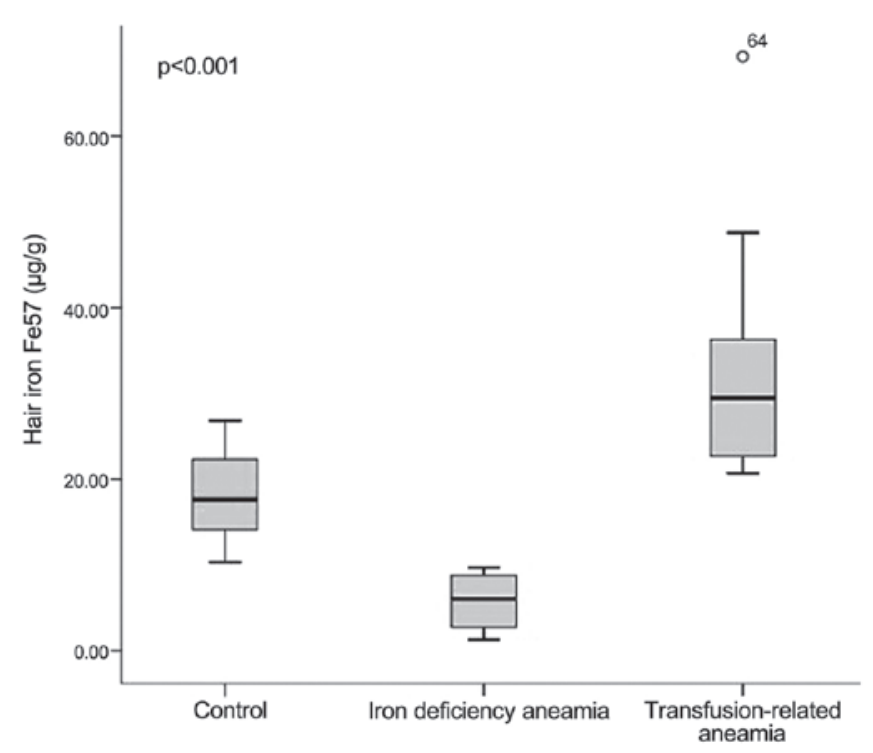

Figure 2. Results of hair iron ${ }^{57} \mathrm{Fe}$ ppm concentration in the groups.

and ${ }^{57} \mathrm{Fe}$ concentratons and TIBC. The results of the correlation analysis are shown in Table II.

\section{Discussion}

Various tests are in current use, including invasive laboratory tests, to show the status of body iron, which has a role in numerous vital functions. In the most widely-used test of serum ferritin level, the body iron status may not be accurately reflected due to various conditions, including pregnancy, acute or chronic inflammatory disease, malignancy, infection, renal failure or malabsorption syndrome.

The hair appears to function as a potential depot for trace elements entering the body. Hair mineral levels are significant 
Table II. Results of the correlation analysis between the parameters.

\begin{tabular}{|c|c|c|c|c|c|c|c|c|c|c|c|c|}
\hline Parameters & 1 & 2 & 3 & 4 & 5 & 6 & 7 & 8 & 9 & 10 & 11 & 12 \\
\hline 1. Hair ${ }^{56} \mathrm{Fe}, \mathrm{ppm}$ & 1 & 0.776 & -0.041 & -0.092 & 0.378 & 0.165 & 0.464 & 0.305 & 0.622 & -0.703 & 0.507 & 0.505 \\
\hline P-value & & $<0.001^{\mathrm{a}}$ & 0.754 & 0.482 & $0.003^{\mathrm{a}}$ & 0.207 & $<0.001^{\mathrm{a}}$ & $0.018^{\mathrm{a}}$ & $<0.001^{\mathrm{a}}$ & $<0.001^{\mathrm{a}}$ & $<0.001^{\mathrm{a}}$ & $<0.001^{\mathrm{a}}$ \\
\hline 2. Hair ${ }^{57} \mathrm{Fe}, \mathrm{ppm}$ & & 1 & 0.177 & 0.102 & 0.522 & 0.371 & 0.571 & 0.170 & 0.642 & -0.759 & 0.473 & 0.494 \\
\hline P-value & & & 0.176 & 0.437 & $<0.001^{\mathrm{a}}$ & $0.005^{\mathrm{a}}$ & $<0.001^{\mathrm{a}}$ & 0.193 & $<0.001^{\mathrm{a}}$ & $<0.001^{\mathrm{a}}$ & $<0.001^{\mathrm{a}}$ & $<0.001^{\mathrm{a}}$ \\
\hline 3. Haemoglobin & & & 1 & 0.969 & 0.705 & 0.754 & 0.615 & -0.304 & 0.069 & -0.160 & -0.112 & -0.127 \\
\hline P-value & & & & $<0.001$ & $<0.001$ & $<0.001$ & $<0.001$ & 0.018 & 0.599 & 0.223 & 0.395 & 0.332 \\
\hline 4. Haematocrit & & & & 1 & 0.612 & 0.631 & 0.537 & -0.278 & -0.015 & -0.066 & -0.184 & -0.210 \\
\hline P-value & & & & & $<0.001$ & $<0.001$ & $<0.001$ & 0.031 & 0.911 & 0.617 & 0.159 & 0.108 \\
\hline 5. $\mathrm{MCH}$ & & & & & 1 & 0.862 & 0.950 & -0.203 & 0.409 & -0.570 & 0.321 & 0.340 \\
\hline P-value & & & & & & $<0.001$ & $<0.001$ & 0.120 & 0.001 & $<0.001$ & 0.015 & 0.008 \\
\hline 6. $\mathrm{MCHC}$ & & & & & & 1 & 0.675 & -0.280 & 0.300 & -0.356 & 0.160 & 0.185 \\
\hline P-value & & & & & & & $<0.001$ & 0.030 & 0.020 & 0.005 & 0.221 & 0.158 \\
\hline 7. MCV & & & & & & & 1 & -0.129 & 0.444 & -0.649 & 0.371 & 0.394 \\
\hline P-value & & & & & & & & 0.325 & $<0.001$ & $<0.001$ & 0.003 & 0.002 \\
\hline 8. RDW & & & & & & & & 1 & 0.224 & -0.228 & 0.310 & 0.261 \\
\hline P-value & & & & & & & & & 0.086 & 0.080 & 0.016 & 0.044 \\
\hline 9. Serum iron & & & & & & & & & 1 & -0.779 & 0.753 & 0.669 \\
\hline $\mathrm{P}$-value & & & & & & & & & & $<0.001$ & $<0.001$ & $<0.001$ \\
\hline 10. TIBC & & & & & & & & & & 1 & -0.812 & -0.687 \\
\hline P-value & & & & & & & & & & & $<0.001$ & $<0.001$ \\
\hline 11. TS & & & & & & & & & & & 1 & 0.606 \\
\hline $\mathrm{P}$-value & & & & & & & & & & & & $<0.001$ \\
\hline 12. Ferritin & & & & & & & & & & & & 1 \\
\hline
\end{tabular}

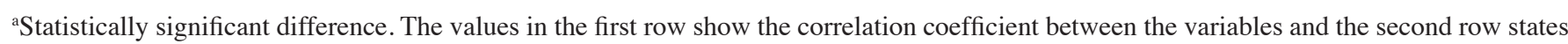
the P-value for the level of statistical significance of these coefficients. $\mathrm{MCH}$, mean erythrocyte haemoglobin; MCHC, mean erythrocyte haemoglobin concentration; MCV, mean erythrocyte volume; RDW, erythrocyte distribution width; TIBC, total iron-binding capacity; TS, transferrin saturation percentage.

in terms of showing the basic composition of minerals accumulated over a long time period. Therefore, hair is a good biopsy material for the accurate evaluation of trace elements. Previous studies have shown that there is a significant correlation between basic elements found in the body and the hair, and in this context hair mineral analysis can be used as a good marker of body mineral levels $(5,6)$. In comparison with other methods investigating body mineral levels, hair mineral analysis has long-term stability and is not immediately affected by changes in mineral intake (7). This is important with respect to being able to accurately reflect the body iron status in several different circumstances. However, the specifications of hair iron concentration necessitate a strict sampling regime and as this is not practical, clinical use is limited.

There are extremely few data associated with the use of hair iron concentration to define body iron status. In a study by Duffield and Green (8) in 1956, it was concluded that hair iron concentration may not provide sufficient information regarding total body iron. In 1971, Lovric and Pepper (9) conducted a study measuring the iron content of various hair segments of children with iron deficiency and iron overload and concluded that there was no significant association between the groups with respect to hair iron concentration. However, in the following years, Bisse et al (1) proposed that hair iron concentration may be useful in the evaluation of body iron status and stated that further studies were required on this subject (1).

In the present study, the difference in body iron content shown between the iron deficiency anaemia group and the transfusion-related anaemia group showed a significant difference with respect to hair iron concentrations. The highest hair iron concentration $(89.41 \mu \mathrm{g} / \mathrm{g})$ of the highest ferritin value was observed in the transfusion-related anaemia patient group and the lowest hair iron concentration $(0.77 \mu \mathrm{g} / \mathrm{g})$ was determined in the iron deficiency anaemia group $(\mathrm{P}<0.001)$. In addition, a positive correlation was determined between hair iron ${ }^{56} \mathrm{Fe}$ and ${ }^{57} \mathrm{Fe}$ concentrations and serum iron, ferritin level, transferrin saturation, $\mathrm{MCV}$ and $\mathrm{MCH}$ values, which are the most important parameters showing body iron content. A statistically significant negative correlation was determined with TIBC.

The factors that can restrict the measurement of hair iron include the diversity shown in scalp and hair samples, different lengths of hair samples that may affect results, the possibility of environmental contamination and that hair iron concentration reference intervals have not been clearly 
defined. Other factors limiting the clinical application of hair iron measurement are that the measurement requires a strict sampling regime and non-invasive magnetic resonance imaging is mainly able to evaluate mineral density in solid tissue. However, it is important that the results of the present study have shown a significant association between body iron content laboratory markers and hair iron concentrations, and thus demonstrate that hair sampling can be used as a marker of body iron content.

As it is not known what type of association there is between the iron content of solid organs and hair iron concentration in cases of increased iron accumulation, there is a requirement for future studies to show how hair iron is affected and what the association is between hair iron concentration and iron levels in other solid organs when ferritin acts as an acute phase reactant.

In conclusion, the present study has shown that patient groups with different body iron content showed a significant difference in hair iron concentration and these values were correlated with laboratory markers of body iron content. These results support the view that hair sampling can be used as a marker of body iron content.

\section{Acknowledgements}

The authors would like to thank Erciyes University for their contribution.

\section{References}

1. Bisse E, Renner F, Sussmann S, Scholmerich J and Wieland H: Hair iron content: possible marker to complement monitoring therapy of iron deficiency in patients with chronic inflammatory bowel diseases? Clin Chem 42: 1270-1274, 1996.

2. El Sayed SM, Abou-Taleb A, Mahmoud HS, Baghdadi H, Maria RA, Ahmed NS and Nabo MM: Percutaneous excretion of iron and ferritin (through Al-hijamah) as a novel treatment for iron overload in beta-thalassemia major, hemochromatosis and sideroblastic anemia. Med Hypotheses 83: 238-246, 2014.

3. Vichinsky E, Butensky E, Fung E, Hudes M, Theil E, Ferrell L, Williams R, Louie L, Lee PD and Harmatz P: Comparison of organ dysfunction in transfused patients with SCD or beta thalassemia. Am J Hematol 80: 70-74, 2005.

4. Angelucci E, Brittenham GM, McLaren CE, Ripalti M, Baronciani D, Giardini C, Galimberti M, Polchi P and Lucarelli G: Hepatic iron concentration and total body iron stores in thalassemia major. N Engl J Med 343: 327-331, 2000.

5. Kedzierska E: Concentrations of selected bioelements and toxic metals and their influence on health status of children and youth residing in Szczecin. Ann Acad Med Stetin 49: 131-143, 2003 (In Polish).

6. Lakshmi Priya MD and Geetha A: Level of trace elements (copper, zinc, magnesium and selenium) and toxic elements (lead and mercury) in the hair and nail of children with autism. Biol Trace Elem Res 142: 148-158, 2011.

7. Ayodele $J$ and Bayero A: Lead and zinc concentrations in hair and nail of some Kano inhabitants. Afr J Env Sci Tech 3: 164-170, 2009.

8. Duffield J and Green PT: The iron content of human hair. II. Individuals with disturbed iron metabolism. Can Serv Med J 12: 987-996, 1956.

9. Lovric VA and Pepper R: Iron content of hair in children in various states of iron balance. Pathology 3: 251-256, 1971. 